\title{
The potential of Ambroxol to be repurposed for the prevention and treatment of respiratory viruses, including influenza viruses, rhinoviruses, coronaviruses, and SARS-CoV-2
}

\author{
Adam Seth Gissen ${ }^{1}$ \\ ${ }^{1}$ Peak Sciences Group (HK) Ltd.
}

April 28, 2020

\begin{abstract}
The emergence in December 2019 of SARS-CoV-2, and its infectious disease COVID-19, has resulted in a worldwide pandemic. Despite the realization that humanity is under a constant threat from respiratory viruses with pandemic potential, such as coronaviruses and influenza viruses, the discovery of a safe and effective prophylactic drug against such infections has remained elusive. The benefits of such a drug for the prevention, containment, or mitigation of emerging and endemic viral threats would be significant. As research on the mechanisms of viral infection has advanced, the repositioning and repurposing of existing drugs and therapeutics has played a significant role in our attempts to find efficacious treatments. This is especially true for COVID19, though numerous existing drugs were previously studied for their usefulness in treating both SARS-CoV and MERS-CoV infections. Ambroxol, a semi-synthetic derivative of the alkaloid vasicine, has been researched and marketed for more than half a century. Ambroxol was originally marketed as a respiratory mucolytic, mucokinetic, expectorant, and secretolytic. Decades of research have shown it to possess multiple effects, with minimal side effects and a favorable safety profile. Among the mechanisms of action and molecular targets that have been shown for Ambroxol, some of these are potentially of benefit for the prevention and treatment of viral infections caused by influenza viruses, rhinoviruses, and coronaviruses. If proven to be safe and effective, the repurposing of Ambroxol as a phylactic and treatment for these viral infections would be a major advancement in public health, by lowering the morbidity and mortality caused by these viruses.
\end{abstract}

\section{Introduction}

Since the epidemic caused by SARS-CoV in 2003 (1), and sporadic cases since 2012 of MERS-CoV (2), there has been concern was that one of these coronaviruses could cause a future epidemic or pandemic. Equally concerning was the potential for a novel and infectious coronavirus to emerge after zoonotic transfer to humans (3). Thus, the discovery that a novel coronavirus was responsible for the unexplained incidences of a SARS-like respiratory syndrome in China in December 2019 was not unexpected. Subsequent to the discovery of this virus, now known as SARS-CoV-2, it was realized that an epidemic caused by this virus was resulting in a new and potentially fatal disease, now named COVID-19 (4). The rapid spread of this new virus, both due to its infectious nature and modern transportation systems, has resulted in a pandemic that is unparalleled in modern history. This pandemic has resulted in an urgent search for effective prevention and treatment strategies. 


\section{Background on Ambroxol (and bromhexine)}

Ambroxol (trans-4-(2-amino-3,5-dibromobenzylamino)-cyclohexanol hydrochloride, CAS 18683-91-5) is used as a mucolytic, mucokinetic, expectorant, and secretolytic drug. It is a metabolite of bromhexine, an older drug marketed for the same uses. Both Ambroxol and bromhexine are semi-synthetic derivatives of vasicine, an alkaloid found in Adhatoda vasica. This plant has a long history in Ayurvedic and Unani traditional medicine as a treatment for a number of diseases and conditions, notably of the respiratory tract (5). Bromhexine was first marketed in 1963 (6). Subsequently, Ambroxol was researched and developed as a drug (7). Although both drugs have been marketed for more than 50 years, early research showed that Ambroxol has higher activity as a bronchosecretolytic (8). Ambroxol was later found to be more effective at inhibiting IgE-dependent mediator secretion from key effector cells involved in allergic inflammation, as well as being less toxic in vitro than bromhexine or vasicine (9). Ambroxol has been more extensively researched in recent years. An assessment of Ambroxol's safety in the treatment of airway diseases in adult patients was published by the European Union's Pharmacovigilance Risk Assessment Committee (PRAC) in 2016, and they concluded that, "Clinical experience accumulated from randomized clinical trials and observational studies suggests that ambroxol is a safe and well-tolerated treatment of bronchopulmonary diseases, with a well-balanced and favorable benefit-risk profile" (10). Marketed in many countries, Ambroxol has been studied and utilized in various forms including syrups, tablets, lozenges, time-release capsules, intravenous, and inhalable presentations.

\section{Ambroxol as a potential phylactic against common viral respiratory diseases}

With its approved use as a treatment for respiratory diseases, it is not surprising that Ambroxol was eventually investigated for its effects on respiratory tract viruses. Up until now, only a small number of such studies have taken place. In 2002, it was shown that Ambroxol had a suppressive effect on influenza A (H3N2) virus proliferation, and improved survival in mice, by increasing the level of antiviral factors (11). Two years later, Ambroxol was studied for its effects on murine-adapted influenza A infection in rats and mice (12). Ambroxol was shown to increase airway fluid levels of secretory leukoprotease inhibitor and pulmonary surfactant, two important components of the mucosal defense system against influenza $\mathrm{A}$ and other respiratory viruses. It also increased the level of immunoglobulin A ( $\operatorname{IgA}$ ). These effects significantly suppressed viral replication, and increased the survival rate of animals given a lethal dose of influenza A virus. The potential use of Ambroxol to increase resistance to influenza A infection, by upregulating the levels of endogenous protease inhibitory compounds that interfere with cellular, trypsin-type, processing proteases that activate the fusion glycoprotein precursors of influenza A virus, was further described by the same researchers in 2007 (13). A year earlier, a study was conducted to determine the effectiveness of long-term use of low dose Ambroxol for one year to prevent Acute Upper Respiratory Disease from influenza and the common cold (14). Although small in size, and complicated by the use of 2 other drugs (rebamipide and carbocisteine) as a comparison to Ambroxol, this study showed a significant reduction in the median number of Acute Upper Respiratory Disease episodes in the Ambroxol group. Another study of Ambroxol on viral respiratory diseases examined its effect on rhinovirus (RV14) infection in primary cultures of human tracheal epithelial cells (15). This study showed that Ambroxol reduced the susceptibility to infection, lowered rhinovirus RV14 titers, reduced cytokine concentrations of interleukin (IL)- $1 \beta$, IL- 6 and IL-8, and reduced the number of acidic endosomes needed for viral RNA entry to the cytoplasm. This outcome was partly ascribed to Ambroxol's ability to modulate airway inflammation, by reducing the activation of the transcription factor nuclear factor kappa $\mathrm{B}(\mathrm{NF}-x \mathrm{~B})$ in the nucleus. These studies appear to be the only investigations of Ambroxol's direct antiviral effects and mechanisms.

\section{Ambroxol as a potential phylactic against SARS-CoV-2 and COVID-19}

Due to the current pandemic of SARS-CoV-2 infections, and COVID-19, there is a desperate need for effective preventative measures and treatments. Ambroxol has demonstrated properties that go beyond its potential ability to prevent infection by enhancing passive mucosal defenses, because it may also be able to directly 
interfere with the SARS-CoV-2 life-cycle as has been demonstrated with influenza A and rhinovirus RV14. Several of these effects may be beneficial to circumvent the progression of SARS-CoV-2 infection to COVID19. A recent preprint paper showed that elevated interleukin-6 (IL-6) was not only strongly associated with the need for mechanical ventilation, but also that IL-6 levels in patients could predict respiratory failure with a high degree of accuracy (16). A meta-analysis in 2014 of studies related to Ambroxol's effects on acute lung injury and respiratory distress syndrome determined that Ambroxol appeared to significantly decrease the production of IL-6 (17). One of the exciting areas of research into the infectious mechanisms of numerous viruses, inclucing SARS-CoV-2, is that of autophagy. A mini-review on autophagy and endocytosis, related to SARS-CoV-2 infection and potential treatments, was published in March 2020 (18). Recent reviews on the functions of autophagy in other viral infections have also been published (19-21). Ambroxol has been researched for its effect on autophagy, under varied conditions. Interestingly, research has shown that Ambroxol can either stimulate or block autophagy. Ambroxol has been shown to increase the lung and airway fluid levels of several different classes of antibiotics, an effect thought to be related to Ambroxol's stimulation of autophagy (22). This property may have relevance for the treatment of secondary infections due to respiratory viruses. In a study utilizing Ambroxol to enhance intracellular levels of the experimental cancer chemotherapeutic "paclitaxel polymer micelles", Ambroxol was shown to inhibit autophagy and promote lung accumulation of the micelles (23). Another recent study examined the effects of Ambroxol on the autophagy-lysosome pathway and mitochondria in neurons (24). The authors suggested that Ambroxol was blocking autophagy, and driving cargo towards the secretory pathway. Mitochondrial content was also found to be increased by ambroxol via peroxisome proliferator-activated receptor gamma coactivator 1alpha (PGC1- $\alpha$ ). The effects of viral infections on organelle dynamics, including mitochondrial function, has been reviewed previously (25). Additionally, the relationship between autophagy, mitochondria, and the inflammasome has also been reviewed (26). These authors described the relationship between autophagy and inflammasome activation, and the central role that mitochondria play in both cellular processes. In the specific case of SARS-CoV, it was found that a protein encoded by SARS-CoV open reading frame9b (ORF-9b) localizes to mitochondria causing several perturbations in their function (27). This research concluded that these results indicated that SARS-CoV ORF-9b negatively influences mitochondrial function, interfering with interferon production, to help bypass host innate immunity. A recent review examined the complex interactions between innate immunity, and the strategies used to evade the immune system by viruses including influenza, rhinoviruses, and coronaviruses (28). These strategies are considered vital for the relationship between host immunity and viral immune evasion. One of the realities of the SARS-CoV-2 pandemic is that advanced age and age-related, underlying health problems such as obesity, heart disease, and diabetes are risk factors for the development for severe COVID-19 (29). The exact reason(s) for this remains to be elucidated. One of the common factors of both advanced age and age-related health problems is autophagy. Defects in the autophagy-lysosomal-mitochondrial axis has been shown to be significantly related to the senescence of cells (30). The administration of Ambroxol has been shown to reverse some of these deficits using an in vitro model of senescence (31). Another common feature related to aging is insulin resistance. This is thought to be partly attributable to defects in autophagy (32). Ambroxol has been shown to significantly reduce insulin resistance, by upregulating neuraminidase 1 , in obese mice (33). The direct effects of Ambroxol on the factors involved in SARS-CoV-2 infection, and COVID-19 development, have yet to be determined.

\section{Ambroxol as a potential phylactic for ventilator-induced lung injury}

One of the serious complications of ventilator use is ventilator-induced lung injury. The cause of this injury is believed to be multi-factorial. Primary mechanisms include a ventilator-induced depression of antioxidant protection, and an increase in inflammatory mediators. A recent study showed that Ambroxol administration could significantly lessen the extent of ventilator-induced injury in rats (34). The proposed mechanisms of action included a decrease in ventilation-induced lung inflammation, and an increase in depressed levels of the antioxidant glutathione. Similar beneficial effects were found in a study published in 2000 that examined the effects of Ambroxol on ventilator-induced lung injury in rats (35). This study showed that Ambroxol lowered 
the production of inflammatory cytokines in the lung, corrected the imbalance of antioxidant protection, and had a beneficial effect on the incidence of severe lung injuries. Conversely, a study looking at the effects of high-dose, oral Ambroxol on pediatric acute respiratory distress syndrome, predominantly from pneumonia and bronchiolitis, found no significant beneficial effect of Ambroxol therapy, though there were also no significant adverse effects (36). In the case of extracorporeal circulation during surgery, Ambroxol was found to inhibit decreases in the quality of pulmonary surfactant (37). Ambroxol has also been studied for its potential to prevent biofilm-dependent airway infections in ventilated patients (38). It was also shown to be effective in preventing biofilm-associated lung infection in a rat model of acute lung infection induced by endotracheal intubation with a tube covered with mucoid P. aeruginosa biofilm (39). This study showed that Ambroxol could destroy the structure of the biofilm on the tube used for intubation. Treatment was also effective at reducing the bacterial load, as well as lowering the cytokine response and protecting the lung from pathological changes caused by the bacterial infection.

\section{Challenges for the study and repurposing of Ambroxol against respiratory viruses}

One of the unique aspects of Ambroxol's use as a drug is the wide range of doses, and variety of presentation forms, that have been used clinically and experimentally. This presents some challenges to proving Ambroxol's value, as well as obtaining potential benefits that could be related to dose or form of administration. Because of the multiple effects possessed by Ambroxol, it will be necessary to determine the dose-related effects to optimize clinical results. As a prophylactic (prephylactic), low doses may be sufficient to enhance mucosal protective factors against respiratory viral infections, potentially lowering their incidence. Larger doses will likely be needed to directly interfere with viral reproduction, or prevent ventilator-induced lung injury. Ambroxol has proven itself to have a favorable safety profile, over a wide dosage range. This should aid in the research and development of potentially useful prevention and treatment regimens.

\section{Conclusion}

Existing evidence suggests that Ambroxol, and possibly bromhexine, may have potential value as an antiviral drug against against influenza A and rhinoviruses. Ambroxol's mechanisms of action also suggest that it may have applications for the treatment of human coronavirus infections, including SARS-CoV-2 and COVID19. Additionally, Ambroxol may possess synergistic or additive effects when combined with drugs currently being used for the treatment of influenza, or investigated for the treatment of COVID-19. However, and importantly, there is currently no clinical or experimental evidence that Ambroxol or bromhexine are safe and effective for the prevention or treatment of SARS-CoV-2 infection and COVID-19. Ambroxol may have the unique ability to be utilized as a prophylactic (prephylactic) to prevent infection by respiratory viruses, due to its well-balanced and favorable benefit-risk profile. The current lack of such a preemptive strategy is a hindrance to lowering the morbidity and mortality from both common and emerging viral respiratory diseases. Hopefully, the existing evidence will encourage further research on Ambroxol and bromhexine for their safety and efficacy as a preventive and treatment for respiratory viral infections.

\section{References}

1. Ksiazek TG, Erdman D, Goldsmith CS, et al. A novel coronavirus associated with severe acute respiratory syndrome. N Engl J Med. 2003 May 15;348(20):1953-66.

2. Zaki AM, van Boheemen S, Bestebroer TM, et al. Isolation of a novel coronavirus from a man with pneumonia in Saudi Arabia. N Engl J Med. 2012 Nov 8;367(19):1814-20.

3. Wang LF, Anderson DE. Viruses in bats and potential spillover to animals and humans. Curr Opin Virol. 2019 Feb;34:79-89. 
4. Zhu N, Zhang D, Wang W, et al. A Novel Coronavirus from Patients with Pneumonia in China, 2019. N Engl J Med. 2020 Feb 20;382(8):727-733.

5. Claeson UP, Malmfors T, Wikman G, et al. Adhatoda vasica: a critical review of ethnopharmacological and toxicological data. J Ethnopharmacol. 2000 Sep;72(1-2):1-20.

6. Zanasi A, Mazzolini M, Kantar A. A reappraisal of the mucoactive activity and clinical efficacy of bromhexine. Multidiscip Respir Med. 2017 Mar 20;12:7.

7. Malerba M, Ragnoli B. Ambroxol in the 21st century: pharmacological and clinical update. Expert Opin Drug Metab Toxicol. 2008 Aug;4(8):1119-29.

8. Wiessmann KJ, Niemeyer K. [Clinical results in the treatment of chronic obstructive bronchitis with ambroxol in comparison with bromhexine (author's transl)]. Arzneimittelforschung. 1978;28(5a):918-21.

9. Gibbs BF. Differential modulation of IgE-dependent activation of human basophils by ambroxol and related secretolytic analogues. Int J Immunopathol Pharmacol. 2009 Oct-Dec;22(4):919-27.

10. Cazan D, Klimek L, Sperl A, et al. Safety of ambroxol in the treatment of airway diseases in adult patients. Expert Opin Drug Saf. 2018 Dec;17(12):1211-1224.

11. Yang B, Yao DF, Ohuchi M, et al. Ambroxol suppresses influenza-virus proliferation in the mouse airway by increasing antiviral factor levels. Eur Respir J. 2002 May;19(5):952-8.

12. Kido H, Okumura Y, Yamada H, et al. Secretory leukoprotease inhibitor and pulmonary surfactant serve as principal defenses against influenza A virus infection in the airway and chemical agents up-regulating their levels may have therapeutic potential. Biol Chem. 2004 Nov;385(11):1029-34.

13. Kido H, Okumura Y, Yamada H, et al. Proteases essential for human influenza virus entry into cells and their inhibitors as potential therapeutic agents. Curr Pharm Des. 2007;13(4):405-14.

14. Nobata K, Fujimura M, Ishiura Y, et al. Ambroxol for the prevention of acute upper respiratory disease. Clin Exp Med. 2006 Jun;6(2):79-83.

15. Yamaya M, Nishimura H, Nadine LK, et al. Ambroxol inhibits rhinovirus infection in primary cultures of human tracheal epithelial cells. Arch Pharm Res. 2014 Apr;37(4):520-9.

16. Tobias Herold III, Vindi Jurinovic, Chiara Arnreich, et al. Level of IL-6 predicts respiratory failure in hospitalized symptomatic COVID-19 patients. medRxiv 2020.04.01.20047381; doi: https://doi.org/10. $1101 / 2020.04 .01 .20047381$

17. Wu X, Li S, Zhang J, et al. Meta-analysis of high doses of ambroxol treatment for acute lung injury/acute respiratory distress syndrome based on randomized controlled trials. J Clin Pharmacol. 2014 Nov;54(11):1199-206.

18. Yang N, Shen HM. Targeting the Endocytic Pathway and Autophagy Process as a Novel Therapeutic Strategy in COVID-19. Int J Biol Sci. 2020 Mar 15;16(10):1724-1731.

19. Wong HH, Sanyal S. Manipulation of autophagy by (+) RNA viruses. Semin Cell Dev Biol. 2019 Aug 8. pii: S1084-9521(18)30222-2.

20. Abdoli A, Alirezaei M, Mehrbod P, et al. Autophagy: The multi-purpose bridge in viral infections and host cells. Rev Med Virol. 2018 Jul;28(4):e1973.

21. Paul P, Münz C. Autophagy and Mammalian Viruses: Roles in Immune Response, Viral Replication, and Beyond. Adv Virus Res. 2016;95:149-95.

22. Deretic V, Timmins GS. Enhancement of lung levels of antibiotics by ambroxol and bromhexine. Expert Opin Drug Metab Toxicol. 2019 Mar;15(3):213-218. 
23. He W, Xiao W, Zhang X, et al. Pulmonary-Affinity Paclitaxel Polymer Micelles in Response to Biological Functions of Ambroxol Enhance Therapeutic Effect on Lung Cancer. Int J Nanomedicine. 2020 Feb 4;15:779793.

24. Magalhaes J, Gegg ME, Migdalska-Richards A, et al. Effects of ambroxol on the autophagy-lysosome pathway and mitochondria in primary cortical neurons. Sci Rep. 2018 Jan 23;8(1):1385.

25. Glingston RS, Deb R, Kumar S, et al. Organelle dynamics and viral infections: at cross roads. Microbes Infect. 2019 Jan - Feb;21(1):20-32.

26. de Lavera I, Pavon AD, Paz MV, et al. The Connections Among Autophagy, Inflammasome and Mitochondria. Curr Drug Targets. 2017;18(9):1030-1038.

27. Shi CS, Qi HY, Boularan C, et al. SARS-coronavirus open reading frame-9b suppresses innate immunity by targeting mitochondria and the MAVS/TRAF3/TRAF6 signalosome. J Immunol. 2014 Sep 15;193(6):3080-9.

28. Kikkert M. Innate Immune Evasion by Human Respiratory RNA Viruses. Innate Immun. 2020;12(1):420.

29. Muniyappa R, Gubbi S. COVID-19 Pandemic, Corona Viruses, and Diabetes Mellitus. Am J Physiol Endocrinol Metab. 2020 Mar 31. doi: 10.1152/ajpendo.00124.2020. [Epub]

30. Park JT, Lee YS, Cho KA, et al. Adjustment of the lysosomal-mitochondrial axis for control of cellular senescence. Ageing Res Rev. 2018 Nov;47:176-182.

31. Tai H, Wang Z, Gong H, et al. Autophagy impairment with lysosomal and mitochondrial dysfunction is an important characteristic of oxidative stress-induced senescence. Autophagy. 2017 Jan 2;13(1):99-113.

32. Shou J, Chen PJ, Xiao WH. Mechanism of increased risk of insulin resistance in aging skeletal muscle. Diabetol Metab Syndr. 2020 Feb 11;12:14.

33. Fougerat A, Pan X, Smutova V, et al. Neuraminidase 1 activates insulin receptor and reverses insulin resistance in obese mice. Mol Metab. 2018 Jun;12:76-88.

34. Cao DW, Hou MX, Zhang XR. Ambroxol alleviates ventilator-induced lung injury by inhibiting c-Jun expression. Eur Rev Med Pharmacol Sci. 2019 Jun;23(11):5004-5011.

35. Zhang B, Liu Y. [Prophylaxis against ventilator-induced lung injury by Ambroxol]. [Article in Chinese] Zhonghua Yi Xue Za Zhi. 2000 Jan;80(1):51-3.

36. Baranwal AK, Murthy AS, Singhi SC. High-dose Oral Ambroxol for Early Treatment of Pulmonary Acute Respiratory Distress Syndrome: an Exploratory, Randomized, Controlled Pilot Trial. J Trop Pediatr. 2015 Oct;61(5):339-50.

37. Marcatili S, Guarino C, Giannattasio A, et al. Alterations of the endoalveolar surfactant after surgery with extracorporeal circulation. Respiration. 1990;57(4):233-8.

38. Cataldi M, Sblendorio V, Leo A, et al. Biofilm-dependent airway infections: a role for ambroxol? Pulm Pharmacol Ther. 2014 Aug;28(2):98-108.

39. Li F, Wang W, Hu L, et al. Effect of ambroxol on pneumonia caused by Pseudomonas aeruginosa with biofilm formation in an endotracheal intubation rat model. Chemotherapy. 2011;57(2):173-80.

For Correspondence:

peaksciencesgroup@gmail.com 
Competing interests: All authors have completed the ICMJE uniform disclosure form at www.icmje.org/coi_disclosure.pdf and declare: no support from any organization for the submitted work; no financial relationships with any organizations that might have an interest in the submitted work in the previous three years; no other relationships or activities that could appear to have influenced the submitted work. 